\title{
AN ASYMPTOTIC OPTIMAL DESIGN ${ }^{1}$
}

\author{
KAMEL REKAB \\ Florida Institute of Technology \\ Department of Applied Mathematics \\ $150 \mathrm{~W}$. University Blvd. \\ Melbourne, Florida 32901, USA
}

\begin{abstract}
The problem of designing an experiment to estimate the product of the means of two normal populations is considered. A Bayesian approach is adopted in which the product of the means is estimated by its posterior mean. A fully sequential design is proposed and shown to be asymptotically optimal.
\end{abstract}

Key words: Bayes risk, martingales, uniform integrability.

AMS (MOS) subject classifications: $\quad 62 \mathrm{~L} 05,62 \mathrm{~L} 10$.

\section{INTRODUCTION}

Given two independent populations $P_{1}$ and $P_{2}$ indexed by unknown parameters $\theta$ and $\omega$ respectively, it is wished to estimate the product $\theta \omega$ based on a fixed total number of observations $N$. The question is how to allocate the $N$ observations between the two populations so as to minimize the expected squared error loss. For estimating the difference of two normal means, Srivastava (1970) and Robbins, Simons and Starr (1967) have proposed a class of sequential rules incorporating both a sampling scheme and a stopping rule which are asymptotically optimal. For estimating a linear function of mean vectors, Mukhopadhyay and Liberman (1989) have proposed various two-stage and sequential procedures. Rekab (1989) proposed a sequential procedure shown to be asymptotically optimal; however, its application is elusive. We are thus led into seeking an easily expressible procedure which is close to the optimal (in the sense of Bayes) risk. The procedure will be proposed in Section 2. Its form resembles in principle that of Robbins et al. (1967), where the setting is classical rather than Bayesian.

A major difficulty in designing a nonlinear experiment is that the performance of design depends on unknown parameters. To utilize the information fully the experiment must be conducted sequentially. The choice of the next design point is determined by the estimate

\footnotetext{
${ }^{1}$ Received: December, $1990 . \quad$ Revised: May, 1991.
} 
of the unknown parameters based on the observations made to date.

To formalize the problem, suppose that $\theta$ and $\omega$ are independent random variables which have (prior) normal distributions, say $\theta \sim N(\mu, 1 / r), \omega \sim N(\nu, 1 / s)$. Given $\theta, \omega$, let $X_{1}, X_{2}, \ldots Y_{1}, Y_{2}, \ldots$ be independent, with $X_{i} \sim \mathrm{N}(\theta, 1)$ and $Y_{i} \sim \mathrm{N}(\omega, 1)$. Let $n_{k}$, $m_{k}$ denote respectively the number of $X^{\prime} s$ and $Y^{\prime} s$ sampled up to stage $k$. Let $\mathcal{F}_{j l}=$ $\sigma\left(X_{1}, \ldots, X_{j} ; Y_{1}, \ldots, Y_{l}\right)$. An allocation rule will be a stochastic process $\mathcal{A}=\left\{\left(n_{k}, m_{k}\right)\right\}_{k \geq 1}$ on $\mathcal{N}^{2}$ that increases $n_{k}$ or $m_{k}$ by 1 at stage $\mathrm{k}$, and satisfies

$$
\left\{\left(n_{k}, m_{k}\right)=(j, l),\left(n_{k+1}, m_{k+1}\right)=(j+1, l)\right\} \in \mathcal{F}_{j l}
$$

for all $k \in \mathcal{N}, j \in \mathcal{N}, l \in \mathcal{N}$. Let $\mathcal{F}_{k}=\mathcal{F}_{k}(\mathcal{A})=\left\{A: A \cap\left\{\left(n_{k}, m_{k}\right)=(j, l)\right\} \in \mathcal{F}_{j, l}, \forall j, l\right\}$. Then it follows that $\mathcal{F}_{k}$ is a canonical filtering. A sequential procedure will be a sequence of allocation rules $\left\{\left(N, \mathcal{A}_{N}\right)\right\}_{N \geq 1}$.

Let $\mu_{j}$ be the posterior mean of $\theta$ given $X_{1}, \ldots, X_{j}$; that is

$$
\mu_{j}=\frac{r \mu+\sum_{i=1}^{j} X_{i}}{j+r}
$$

for $j=1, \ldots$. Similarly let $\nu_{j}$ be the posterior mean of $\omega$ given $Y_{1}, \ldots, Y_{j}$; that is

$$
\nu_{j}=\frac{s \nu+\sum_{i=1}^{j} Y_{i}}{j+s}
$$

for $j=1, \ldots$ Then

$$
\theta, \omega \mid \mathcal{F}_{k} \sim N\left(\mu_{n_{k}}, \frac{1}{n_{k}+r}\right) \times N\left(\nu_{m_{k}}, \frac{1}{m_{k}+s}\right)
$$

The study proceeds until stage $N$ (fixed). To simplify the notation, $n_{N}$ and $m_{N}$ are denoted by $n$ and $m$. Now consider the problem of estimating the product $\theta \omega$ with squared error loss. It is well known that the Bayes risk is minimized by taking the posterior means as estimates. Then

$$
\mathcal{R}(\mathcal{P})=E\left(\frac{\mu_{n}^{2}}{m+s}+\frac{\nu_{m}^{2}}{n+r}+\frac{1}{(n+r)(m+s)}\right) .
$$

Rekab (1990) derived the following result:

$$
\mathcal{R}(\mathcal{P}) \geq \frac{E(|\theta|+|\omega|)^{2}}{N+r+s}+o(1 / N)
$$


as $N \rightarrow+\infty$ with equality if and only if the following three conditions are satisfied:

(i) $m, n \rightarrow+\infty$ in probability as $N \rightarrow+\infty$

(ii) $\frac{m}{N} \rightarrow \frac{|\theta|}{|\theta|+|\omega|}$ in probability as $N \rightarrow+\infty$

(iii) $\mu_{n}^{2} \frac{N}{m}$ and $\quad \nu_{m}^{2} \frac{N}{n}$ are uniformly integrable.

In the next section an easily expressible procedure is proposed.

\section{THE PROCEDURE.}

One way of solving the problem is to estimate the unknown parameters $\theta$ and $\omega$ at each stage. A procedure of this kind is referred to as the fully sequential procedure. To derive the fully sequential procedure $\mathcal{P}^{*}$, observe that for all $k$

$$
\begin{aligned}
\mathcal{R}(\mathcal{P}) & =E\left\{\frac{\mu_{n_{k}}^{2}}{m_{k}+s}+\frac{\nu_{m_{k}}^{2}}{n_{k}+r}+\frac{1}{\left(n_{k}+r\right)\left(m_{k}+s\right)}\right\} \\
& =E\left\{\frac{\left(\left|\mu_{n_{k}}\right|+\left|\nu_{m_{k}}\right|\right)^{2}}{k+r+s}\right\} \\
& +E\left\{\frac{\left(\left(m_{k}+s\right)\left|\nu_{m_{k}}\right|-\left(n_{k}+r\right)\left|\mu_{n_{k}}\right|\right)^{2}}{(k+r+s)\left(m_{k}+s\right)\left(n_{k}+r\right)}\right\} \\
& +E\left\{\frac{1}{\left(m_{k}+s\right)\left(n_{k}+r\right)}\right\} .
\end{aligned}
$$

Since $\mu_{n_{k}}$ and $\nu_{m_{k}}$ are uniformly integrable martingales, one could minimize the above equality by setting

$$
\frac{m_{k}+s}{n_{k}+r}=\frac{\left|\mu_{n_{k}}\right|}{\left|\nu_{m_{k}}\right|}
$$

With a motivation to move $\frac{m_{k}+s}{n_{k}+r}$ toward the ideal value $\frac{\left|\mu_{n_{k}}\right|}{\left|\nu_{m_{k}}\right|}$, one may define a sequential procedure $\mathcal{P}^{*}$ as follows: Start by taking one observation from each population. Then at stage $k+1$ choose $Y$ if

$$
\frac{m_{k}+s}{n_{k}+r}<\frac{\left|\mu_{n_{k}}\right|}{\left|\nu_{m_{k}}\right|}
$$

and $X$ otherwise.

Theorem 2.1 Let $\mathcal{P}^{*}$ be defined as above. Then

$$
\mathcal{R}\left(\mathcal{P}^{*}\right)=\frac{E(|\theta|+|\omega|)^{2}}{N+r+s}+o(1 / N)
$$


as $N \rightarrow \infty$.

\section{PROOF OF THEOREM 2.1}

As it was mentioned previously, one needs to show that the three conditions listed in Section 1 are verified.

Proof: To show condition (i), note that the proposed sequential procedure can be rewritten as follows: choose $Y$ at stage $k+1$ if

$$
\left(m_{k}+s\right)\left(\frac{\left|\nu_{m_{k}}\right|}{\left|\mu_{n_{k}}\right|+\left|\nu_{m_{k}}\right|}\right)<\left(n_{k}+r\right)\left(\frac{\left|\mu_{n_{k}}\right|}{\left|\mu_{n_{k}}\right|+\left|\mu_{m_{k}}\right|}\right) .
$$

Suppose that $n_{k}$ is bounded. Then the right hand side of (3.1) is bounded. On the other hand, since $k \rightarrow \infty$, the left hand side of (3.1) goes to infinity with probability one. Hence, we have a contradiction. By the same argument it follows that $m_{k} \rightarrow \infty$ almost surely as $k \rightarrow+\infty$. To show condition (ii), let

$$
\begin{aligned}
& k^{\prime}=\sup \left\{i<k: \frac{m_{i}+s}{n_{i}+r}<\frac{\left|\mu_{n_{i}}\right|}{\mid \nu_{m_{i}}}\right\} \\
& k^{\prime \prime}=\sup \left\{i<k: \frac{m_{i}+s}{n_{i}+r} \geq \frac{\left|\mu_{n_{i}}\right|}{\mid \nu_{m_{i}}}\right\} .
\end{aligned}
$$

Then $k^{\prime}, k^{\prime \prime} \rightarrow+\infty$ as $k \rightarrow+\infty$. Now

$$
\begin{aligned}
\frac{m_{k}+s}{n_{k}+r} & =\frac{m_{k^{\prime}}+s}{n_{k}+r}+\frac{1}{n_{k}+r} \leq \frac{m_{k^{\prime}}+s}{n_{k^{\prime}}+r}+\frac{1}{n_{k}+r} \\
& \leq \frac{\left|\mu_{n_{k^{\prime}}}\right|}{\left|\nu_{m_{k^{\prime}}}\right|}+\frac{1}{n_{k}+r}
\end{aligned}
$$

Similarly,

$$
\begin{aligned}
\frac{m_{k}+s}{n_{k}+r} & =\frac{m_{k}+s}{n_{k^{\prime \prime}}+r+1} \geq \frac{m_{k^{\prime \prime}}+s}{n_{k^{\prime \prime}}+r+1} \\
& =\frac{m_{k^{\prime \prime}}+s}{n_{k^{\prime \prime}}+r}\left(1-\frac{1}{n_{k^{\prime \prime}}+r+1}\right) \\
& \geq \frac{\left|\mu_{n_{k^{\prime \prime}}}\right|}{\left|\nu_{m_{k^{\prime \prime}}}\right|}\left(1-\frac{1}{n_{k^{\prime \prime}}+r+1}\right) .
\end{aligned}
$$

Condition (ii) follows easily by letting $k$ go to infinity, since $\mu_{n_{k}} \rightarrow \theta$ and $\nu_{m_{k}} \rightarrow \omega$ by the martingale convergence theorem. Since

$$
\frac{k+r+s}{n_{k}+r} \leq \frac{\left|\mu_{n_{k^{\prime}}}\right|}{\left|\nu_{m_{k^{\prime}}}\right|}+2
$$


it follows that

$$
\frac{N+r+s}{n+r} \nu_{m}^{2} \leq \max _{k}\left|\mu_{n_{k}} \nu_{m_{k}}\right|+2 \nu_{m}^{2} .
$$

Uniform integrability of $\frac{N}{n} \nu_{m}{ }^{2}$ will follow if we establish

$$
\sup _{N} E\left\{\left(\max _{k}\left|\mu_{n_{k}} \nu_{m_{k}}\right|\right)^{\alpha}\right\}<\infty \quad \text { for some } \quad \alpha>1
$$

Since $\mu_{n_{k}} \nu_{m_{k}}=E\left(\theta \omega \mid \mathcal{F}_{k}\right)$, then $\left|\mu_{n_{k}} \nu_{m_{k}}\right|$ is a submartingale. By Doob's inequality for nonnegative submartingales,

$$
\sup _{N} E\left\{\left(\max _{k}\left|\mu_{n_{k}} \nu_{m_{k}}\right|\right)^{2} \leq 4 E(\theta \omega)^{2} .\right.
$$

Thus, uniform integrability of $\frac{N}{n} \nu_{m}{ }^{2}$ is established. A completely analogous argument for $\frac{N+r+s}{m+s} \mu_{n}^{2}$ yields the theorem.

\section{ACKNOWLEDGMENT}

I am grateful to the referee for many helpful comments and remarks, which led to an improvement of an earlier version of this paper.

\section{BIBLIOGRAPHY}

[1] Doob, J. (1953). Stochastic Processes. John Wiley \& Sons, Inc. New York.

[2] Ford, I. and Silvey, S. D. (1980). A sequentially constructed design for estimating a nonlinear parametric function. Biometrika. 67, 381-388.

[3] Mukhopadhyay, N. and Liberman, S. (1989). Sequential estimation of a linear function of mean vectors. Sequential Analysis. 8, 381-394.

[4] Rekab, K. (1989). Asymptotic efficiency in sequential designs. Sequential Analysis. 8, 269-280.

[5] Rekab, K. (1990). Asymptotic optimality of experimental designs in estimating a product of means. J.A.M.S.A. 3, 15-25 .

[6] Robbins, H., Simons, G., and Starr, N. (1967). A sequential analogue of the BehrensFisher problem. Ann. Math. Stat. 38, 1384-1388.

[7] Srivastava, M.S. (1970). On the sequential analogue of the Behrens-Fisher problem. $J$. Roy. Statist. Soc., B 32, 144-148. 


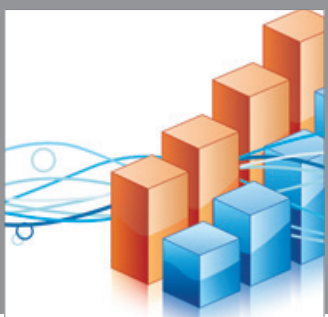

Advances in

Operations Research

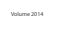

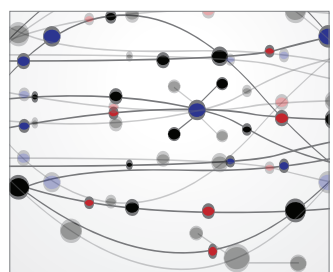

\section{The Scientific} World Journal
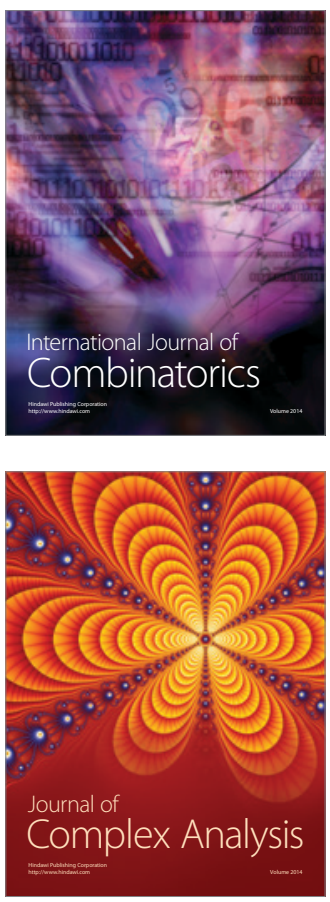

International Journal of

Mathematics and

Mathematical

Sciences
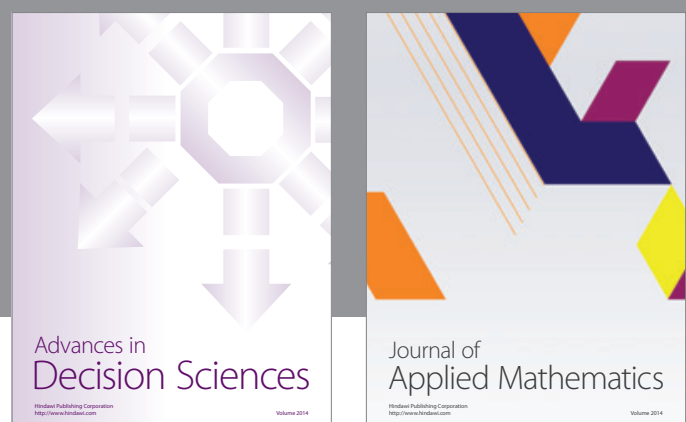

Journal of

Applied Mathematics
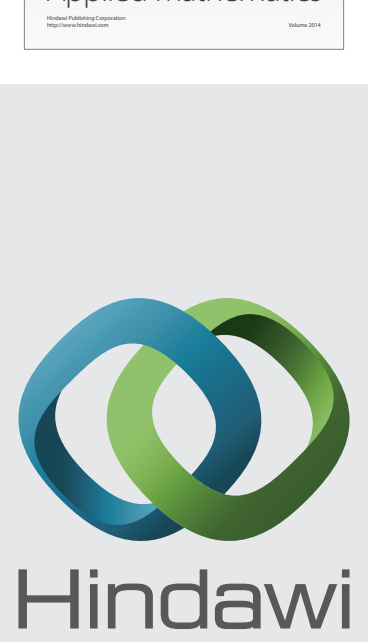

Submit your manuscripts at http://www.hindawi.com
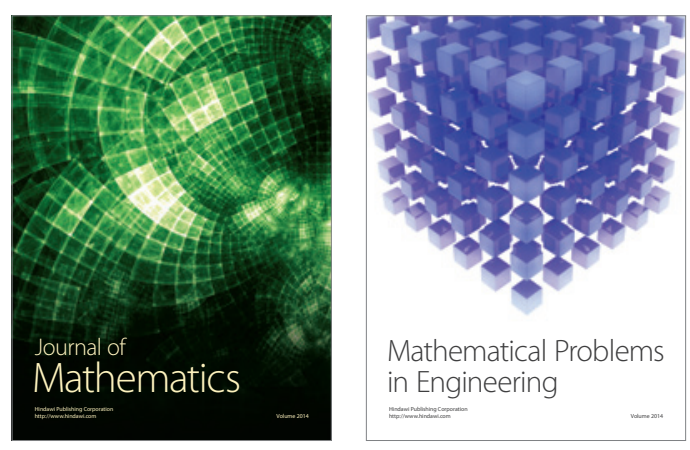

Mathematical Problems in Engineering
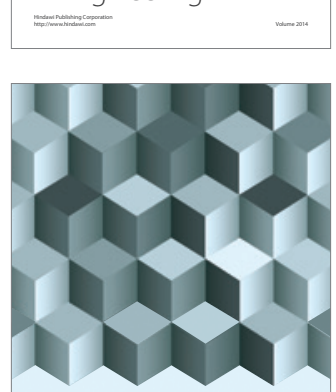

Journal of

Function Spaces
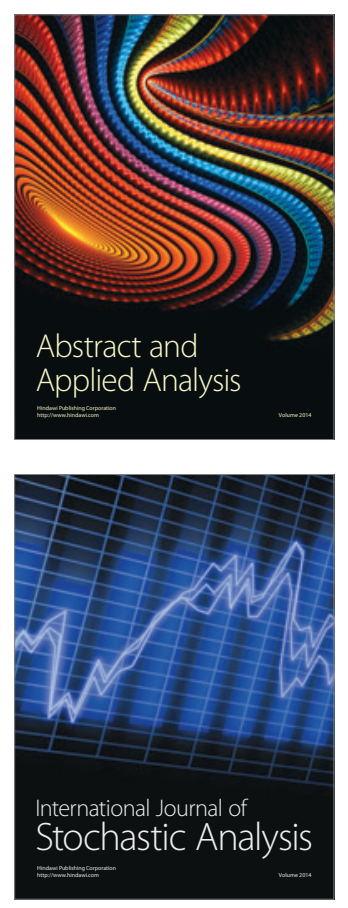

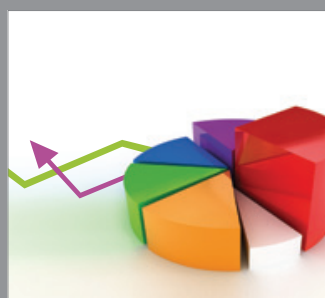

ournal of

Probability and Statistics

Promensencen
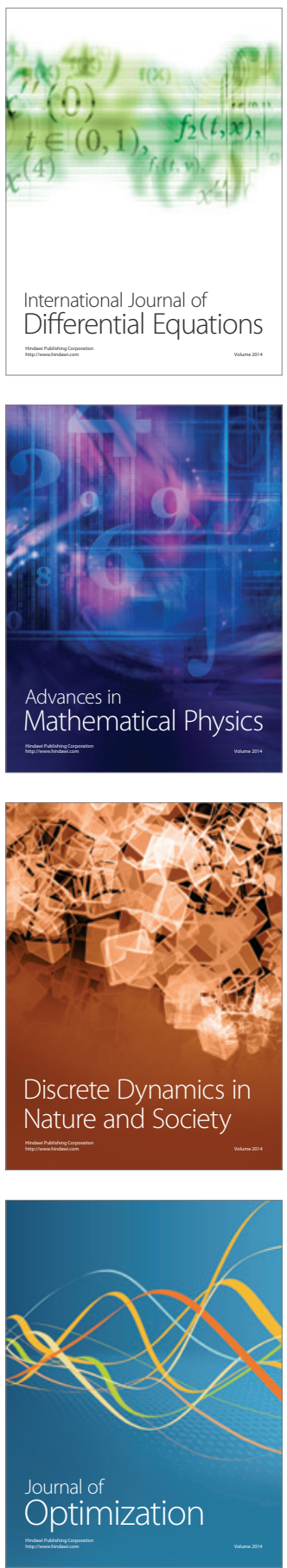